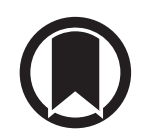

CrossMark

\title{
Preliminary validation of the NTM Module: a patient-reported outcome measure for patients with pulmonary nontuberculous mycobacterial disease
}

\author{
Emily Henkle (1) ${ }^{1}$, Kevin L. Winthrop ${ }^{1}$, Gregory P. Ranches ${ }^{2}$, Wesley Plinke ${ }^{1}$, \\ Hana K. Litvin ${ }^{1}$ and Alexandra L. Quittner ${ }^{3}$
}

Affiliations: ${ }^{1}$ OHSU-PSU School of Public Health, Portland, OR, USA. ${ }^{2}$ Division of Pulmonary and Critical Care Medicine, OHSU, Portland, OR, USA. ${ }^{3}$ Nicklaus Children's Research Institute, Nicklaus Children's Hospital, Miami, FL, USA.

Correspondence: Emily Henkle, 3181 SW Sam Jackson Park Road, Mail Code GH104, Portland, OR 97239, USA. E-mail henkledohsu.edu

\section{@ERSpublications}

The NTM Symptom Module is a valid patient-reported outcome tool that can facilitate patientcentred care and may be used as an outcome in clinical trials to support labelling claims for regulatory bodies. http://bit.ly/2nwlPgi

Cite this article as: Henkle E, Winthrop KL, Ranches GP, et al. Preliminary validation of the NTM Module: a patient-reported outcome measure for patients with pulmonary nontuberculous mycobacterial disease. Eur Respir J 2020; 55: 1901300 [https://doi.org/10.1183/13993003.01300-2019].

\section{ABSTRACT}

Introduction: Nontuberculous mycobacteria (NTM) cause chronic, debilitating pulmonary disease. Patient-reported outcomes provide measures of symptoms, functioning and treatment response. Here we describe the preliminary validation of the recently developed NTM Module.

Methods: The study population included Northwest NTM Biobank patients in whom Mycobacterium avium complex (MAC) was isolated and who had ever met the 2007 American Thoracic Society/Infectious Diseases Society of America pulmonary disease criteria. The NTM Module was administered at enrolment and 12 months; a subset also completed the Quality of Life Questionnaire-Bronchiectasis (QOL-B). The NTM Module generates four domain scores ( $0-100$; higher scores indicate better functioning) reflecting NTM-specific symptoms (NTM Symptoms, Body Image, Digestive Symptoms and Eating Problems). We described patient characteristics and mean scores, and evaluated psychometric properties, including response to treatment at 12 months, for each domain.

Results: Overall, 203 patients with pulmonary MAC disease were included. Average enrolment scores ranged from 76 (NTM Symptoms) to 84 (Eating Problems). Ceiling effects were observed for Body Image (26\% of participants) and Eating Problems (52\%). Internal consistency (Cronbach's alpha) ranged from 0.67 (Digestive Symptoms) to 0.89 (Eating Problems). The intraclass correlation for test-retest reproducibility $(\mathrm{n}=27)$ ranged from 0.72 (Body Image) to 0.94 (Eating Problems). Patients starting treatment $(\mathrm{n}=35)$ had statistically significant increases in scores for NTM Symptoms $(+5, \mathrm{p}=0.04)$, Digestive Symptoms ( $+7, \mathrm{p}=0.002)$, Body Image $(+7, \mathrm{p}=0.03)$ and QOL-B Respiratory Symptoms $(\mathrm{n}=25$, $+10, \mathrm{p}=0.006)$. NTM Symptoms scores increased by 15 points $(\mathrm{p}=0.002)$ in the 16 patients with scores $\leqslant 80$ at enrolment.

Conclusion: The NTM Module generally performs well as a valid patient-reported outcome for pulmonary MAC disease and was responsive to MAC treatment. 


\section{Introduction}

Nontuberculous mycobacteria (NTM) cause chronic, debilitating pulmonary disease. Pulmonary NTM disease is characterised by respiratory symptoms including persistent cough, shortness of breath, fatigue, sputum production and systemic symptoms including low-grade fever, night sweats and weight loss [1]. In the USA, most patients with pulmonary NTM disease also have underlying bronchiectasis, a chronic, inflammatory airway disease, with or without cystic fibrosis (CF) [2]. When treatment is initiated for progressive NTM disease and worsening symptoms, it consists of 18-24 months of a multidrug antibiotic regimen [1]. There are increasing efforts to develop new and improved treatment regimens for NTM disease, and the validation and use of patient-reported outcome (PRO) measures in clinical trials has been identified as a top research priority by NTM patients [3]. PRO measures provide a systematic, standardised assessment of key symptoms and daily functioning from the patient's perspective [4]. Validated PROs may be accepted by the Food and Drug Administration (FDA) for use in product development to support labelling claims [5]. PROs directly measure treatment benefits by capturing improvements in how a patient functions and feels as a result of treatment. In addition, PROs facilitate patient-centred care, better patient-provider communication and the potential for shared decision-making.

In collaboration with patients and NTM experts, Quittner and colleagues developed a PRO for NTM symptoms to compliment the Quality of Life Questionnaire-Bronchiectasis (QOL-B) or Cystic Fibrosis Questionnaire-Revised (CFQ-R) in NTM patients without and with CF, respectively [6-8]. The QOL-B and CFQ-R measure respiratory symptoms in addition to other symptoms and functioning domains. The new NTM PRO was developed following the FDA Guidance on PROs (2009), which requires patient and broader stakeholder input [5]. Here we report the psychometric properties of the NTM Module in a large cohort of patients with pulmonary NTM disease caused by Mycobacterium avium complex (MAC).

\section{Methods}

Study population and data collection

The Northwest NTM Biobank enrols patients with a positive NTM culture, regardless of disease stage or duration. Patients were identified through statewide laboratory surveillance (2013-2016) and through ongoing recruitment at the regional referral clinic at Oregon Health and Science University (2013present). At enrolment and follow-up (12 months later), each patient completed the NTM Module on paper, and a subset also completed the previously validated QOL-B after it was added to the study [8]. We reviewed medical charts at baseline and follow-up and collected a complete clinical history, including NTM disease status, acid-fast bacillus (AFB) smear and culture results, and treatment. The study population was limited to patients with completed chart reviews who had ever met the 2007 American Thoracic Society/Infectious Diseases Society of America (ATS/IDSA) pulmonary disease criteria for MAC without CF [1]. To include a broad spectrum of disease stages and status for psychometric analyses, we did not limit participants to recently diagnosed patients. NTM patients were classified as "cavitary" if the presence of cavities $(\geqslant 2 \mathrm{~cm})$ was indicated on radiologist report from computed tomography (CT) scan; "bronchiectatic" if bronchiectasis was present on CT; "mixed" if patients had both cavities and bronchiectasis; and "unclassified" if they did not have evidence of bronchiectasis or cavitary disease. "Treated COPD" was defined as a diagnosis and current use of oxygen or any inhaled corticosteroid, anticholinergic or long-acting $\beta$-agonist. For the subset with data available for longitudinal analysis of responsivity to NTM treatment, patients were grouped by treatment status at time of enrolment: starting multidrug therapy within 1 week ("starting-treatment") or already on multidrug therapy for at least 90 days ("on-treatment").

\section{NTM Module development}

As previously described, NTM literature was reviewed, and focus groups with both patients and pulmonary experts in NTM were led by a psychologist (A.L. Quittner). Audiotaped transcripts and interviews were coded to identify frequent and impactful symptoms [7]. The NTM Module (table 1) evaluates 1-week recall of symptoms specific to NTM (NTM Symptoms domain: seven items) and three additional domains that arose in the qualitative data collection phase with patients (Body Image: four items, Digestive Symptoms: six items and Eating Problems: three items). The NTM Symptoms domain includes the following items: feverish (chills, sweating), sensitivity to smell, sensitivity to taste, memory problems, bad taste in mouth, bothered by cold weather, problems sleeping. Responses are measured using a four-point Likert scale (e.g. "a lot", "a moderate amount", "a little" or "not at all"). Individual domain scores are calculated, scaled from 0 to 100 (where 100 equates to best health-related quality of life (HRQoL)).The NTM Module was designed to complement the QOL-B, which covers eight additional independent symptom and functioning domains: Respiratory Symptoms, Physical Functioning, Vitality, Role Functioning, Emotional Functioning, Social Functioning, Health Perceptions and (bronchiectasis) Treatment Burden [8]. 
TABLE 1 NTM Module domains and example items

NTM Module Items included (1-week recall)
domains

\section{NTM Symptoms \\ Digestive Symptoms \\ Body Image \\ Eating Problems}

\author{
Feverishness (chills, sweating), sensitivity to smell, sensitivity to taste, memory problems, bad taste in mouth, \\ bothered by cold weather, problems sleeping \\ Constipated, acid reflux, bloating, gas, diarrhoea, abdominal pain \\ Too thin, trouble gaining weight, look different from others, feel bad about physical appearance \\ Force self to eat, eating problems, how feel about eating
}

NTM: nontuberculous mycobacteria.

\section{Statistical analysis}

We described demographic (age, sex) and clinical characteristics (e.g. underlying COPD, pulmonary function testing results, smoking history, bronchiectasis diagnosis). NTM characteristics included cavitary or non-cavitary disease, treatment status, and the most recent AFB smear and culture results. We performed psychometric analyses, including means, standard deviations, item distributions, floor and ceiling effects, and internal consistency (Cronbach's alpha reliability coefficients) for each of the four domains. Our target range for Cronbach's alpha was 0.70 , with a maximum of 0.90 to avoid redundancy of items $[9,10]$. Test-retest reliability was calculated using intraclass correlation coefficients (ICCs) on a subset of patients who completed a second administration $14 \pm 1$ days later. ICC values of $0.50-0.75$ are considered "moderate" and $>0.75$ are considered "excellent" [11, 12].

We analysed factors associated with enrolment scores in a multivariable linear regression model, including pre-specified variables: age $\geqslant 70$, sex, bronchiectasis diagnosis, treated COPD, presence of cavitary disease, most recent AFB culture results, and whether patients were currently on treatment or not. In patients with "active" disease, defined as AFB culture positive within 90 days of enrolment, we evaluated the discriminant validity of each symptom scale using two measures of disease severity (cavitation and AFB smear positivity) as well as underlying lung function (forced expiratory volume in $1 \mathrm{~s}\left(\mathrm{FEV}_{1}\right)$ and forced vital capacity (FVC) \% predicted from pulmonary function testing during the prior year). In addition, we compared enrolment scores for two groups of MAC patients at extremes of disease activity: those with "prior" MAC disease (prior treatment and culture negative at enrolment) and those with "active" MAC disease (never treated and culture positive at enrolment). Comparisons were analysed using the t-test. Responsivity to treatment was calculated as the mean change (difference in score) 12 months after starting MAC therapy, with the within-patient change analysed using the paired t-test. Using a threshold of $\leqslant 80$ for "poor" NTM Symptoms and $\leqslant 70$ for "poor" Respiratory Symptoms, we computed mean score difference for the subset of patients with "poor" enrolment scores in each respective domain.

\section{Results}

Within the Northwest NTM Biobank, we identified 249 pulmonary MAC patients (figure 1) with no CF diagnosis, including 213 (85.5\%) who had ever met the 2007 ATS/IDSA disease criteria. Of these, 203 (95.3\%) patients had NTM Module administered at enrolment and were included in psychometric analyses (table 2). Patients were primarily female $(n=155,76.4 \%)$, mean age was 68.6 years, and the majority had a bronchiectasis diagnosis or CT scan confirmation $(n=167,82.3 \%)$. Fewer were on treatment for COPD $(\mathrm{n}=52,25.6 \%) ; 36(17.7 \%)$ had cavitary disease, and $86(42.4 \%)$ were taking multidrug therapy for their NTM disease. The treated subsets for longitudinal follow-up were similar to each other, except those enrolled on treatment $(n=41)$ were younger (mean age 63.9 versus 69.1 in the $n=35$ starting treatment, $\mathrm{p}=0.06$ ) and less likely to be culture positive (91\% versus $59 \%$ starting treatment, $\mathrm{p}<0.01)$. Among starting-treatment and on-treatment patients, 32 and 41 completed the NTM Module and 21 and 16 completed the QOL-B, respectively.

\section{Mean scores, floor and ceiling effects, internal consistency}

Average domain scores (0-100, with higher scores indicating better functioning) ranged from 76 for NTM Symptoms to 84 for Eating Problems (table 3). Very few patients scored 0 (floor effect), but $26 \%$ of participants scored 100 (ceiling effect) on the Body Image scale and 52\% scored 100 on the Eating Problems scale. Internal consistency was very good for NTM Symptoms (Cronbach's alpha 0.79), Body Image (0.74) and Eating Problems (0.89), and adequate for Digestive Symptoms (0.67). The ICC for testretest reproducibility in 27 patients ranged from 0.72 (Body Image) to 0.94 (Eating Problems), indicating excellent reproducibility over two weeks. 
FIGURE 1 Northwest NTM Biobank patient source population and inclusion flow diagram lincluded for psychometric analysis $n=203$; longitudinal analysis starting treatment $\mathrm{n}=35$, on treatment $\mathrm{n}=411$. MAC: Mycobacterium avium complex; ATS/IDSA: American Thoracic Society/Infectious Diseases Society of America; NTM: nontuberculous mycobacteria.

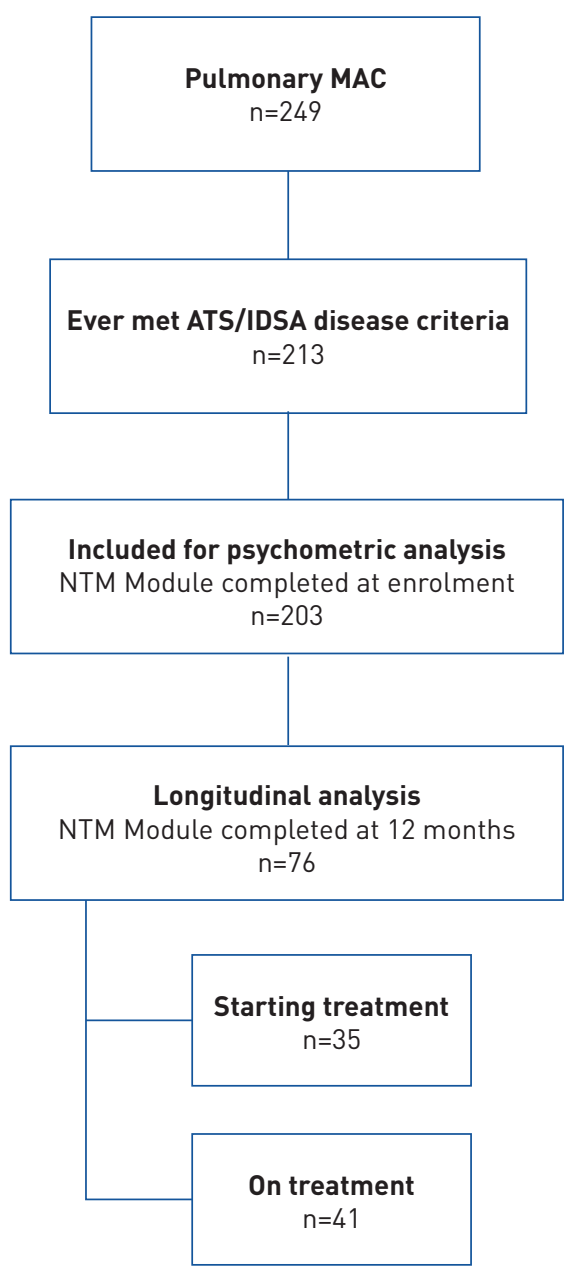

\section{Discriminant validity}

In a multivariable linear model of factors associated with baseline NTM Module scores (table 4), COPD was significantly associated with lower scores across all NTM Module domains (range -6.6 to -11.5 points compared to no COPD). Age, sex and underlying bronchiectasis were not significantly associated with NTM Module scores, nor was the most recent AFB culture positive. The presence of cavitary disease was associated with lower Eating Problems $(-17.0$ points compared to no cavitary disease, $\mathrm{p}<0.001)$ and Body Image $(-11.5, \mathrm{p}=0.03)$. Next, we compared characteristics and scores between groups with varying MAC disease presentation and activity (table 5$)$. Patients with cavitary disease $(\mathrm{n}=32)$ were significantly different from those without cavitary disease $(\mathrm{n}=120)$ : a lower proportion were female (59.4 versus 84.2, $\mathrm{p}=0.002$ ) and had bronchiectasis (65.6 versus $85.8, \mathrm{p}=0.009)$, whereas a higher proportion had treated COPD (40.6 versus 25.0, $\mathrm{p}=0.04$ ) and were on treatment ( 75.0 versus $27.5, \mathrm{p}<0.001)$ (data not shown). Patients with cavitary disease generally had lower NTM Module scores than those with non-cavitary disease, except that Digestive Symptoms scores were similar in the two populations. Eating Problems score was significantly lower (68.9 with cavitary versus 86.7 with non-cavitary disease, $\mathrm{p}=0.002$ ). Despite similar characteristics, patients with active MAC disease $(n=43)$ reported significantly worse Body Image $(72.2$ versus 86.4, $\mathrm{p}=0.007)$ and Eating Problems (81.9 versus 96.7, $\mathrm{p}<0.0001$ ) compared to the group with prior MAC disease $(n=17)$. Further, there were no differences in NTM Module domain scores comparing AFB smear positive $(n=7)$ to smear negative $(n=12)$. Neither $F_{1} V_{1}$ nor FVC were correlated with NTM Symptoms ( $\mathrm{r}=0.14, \mathrm{p}=0.19$ and $\mathrm{r}=0.03, \mathrm{p}=0.80$, respectively), but both were correlated with Respiratory Symptoms $(\mathrm{r}=0.55, \mathrm{p}<0.0001$ and $\mathrm{r}=0.43, \mathrm{p}=0.0014)$.

\section{Responsiveness to treatment}

The mean enrolment scores for the subset starting MAC treatment were $73.9 \pm 16.7$ for NTM Symptoms and ranged from 72 to 82 for the other three domains (table 6). Those starting treatment had statistically significant improvements in NTM Symptoms $(+5.2, \mathrm{p}=0.04)$, Digestive Symptoms $(+6.9, \mathrm{p}=0.002)$ and Body Image $(+7.3, \mathrm{p}=0.03)$ (figure $2 \mathrm{a}$ ). A similar pattern was observed in the subset with QOL-B scores, 
TABLE 2 Characteristics of included patients who met American Thoracic Society/Infectious Diseases Society of America disease criteria for pulmonary Mycobacterium avium complex (MAC) and completed NTM Module and subset available for longitudinal evaluation

\begin{tabular}{|c|c|c|c|}
\hline Characteristics & MAC included & Starting treatment & On treatment \\
\hline Subjects $n$ & 203 & 35 & 41 \\
\hline Female & $155(76.4)$ & $27(77.1)$ & $30(73.2)$ \\
\hline Age years & $68.6 \pm 12.2$ & $69.1 \pm 10.3$ & $63.9 \pm 13.3$ \\
\hline Smoking history ever & $95(46.8)$ & $20(57.1)$ & $14(34.2)$ \\
\hline Treated COPD & $52(27.6)$ & 12 (35.3) & $11(26.8)$ \\
\hline Bronchiectasis & $167(82.3)$ & $29(82.9)$ & 35 (85.4) \\
\hline Pulmonary function testing prior 12 months & 82 (40.3) & $26(74.3)$ & $30(73.1)$ \\
\hline $\mathrm{FEV}_{1} \%$ pred & $71.6 \pm 21.0$ & $70.8 \pm 23.3$ & $71.2 \pm 19.9$ \\
\hline FVC \% pred & $84.2 \pm 17.6$ & $81.1 \pm 21.4$ & $84.7 \pm 14.8$ \\
\hline \multicolumn{4}{|l|}{ Radiographic phenotype } \\
\hline Cavitary & $13(6.4)$ & $2(5.7)$ & $4(9.8)$ \\
\hline Bronchiectatic & $144(70.9)$ & $24(68.6)$ & $31(75.6)$ \\
\hline Mixed cavitary/bronchiectatic & 23 (11.3) & 5 (14.3) & $4(9.8)$ \\
\hline Unclassified & $23(11.3)$ & $4(11.4)$ & $2(4.9)$ \\
\hline Last AFB culture positive & $152(74.9)$ & $32(91.4)$ & $24(58.5)$ \\
\hline Currently meet case definition & $170(83.7)$ & $34(97.1)$ & $41(100.0)$ \\
\hline \multicolumn{4}{|l|}{ Treatment status } \\
\hline Never treated & 68 (33.5) & $27(77.1)$ & \\
\hline Prior completed treatment & $49(24.1)$ & $8(22.9)$ & \\
\hline On treatment & $86(42.4)$ & & $41(100.0)$ \\
\hline Duration of treatment months & $6.3 \pm 7.1$ & & $9.9 \pm 1.5$ \\
\hline
\end{tabular}

Data are presented as $\mathrm{n}(\%)$ or mean $\pm \mathrm{SD}$. $\mathrm{FEV}_{1}$ : forced expiratory volume in $1 \mathrm{~s}$; FVC: forced vital capacity; AFB: acid-fast bacillus. Bold type indicates $p<0.05$ comparing starting-treatment to on-treatment groups.

TABLE 3 NTM Module psychometric analysis results for patients with pulmonary Mycobacterium avium complex disease

\begin{tabular}{lccccc} 
Module domain & Mean scores & $\begin{array}{c}\text { Floor effect } \\
\text { (score=0) }\end{array}$ & $\begin{array}{c}\text { Ceiling effect } \\
\text { (score=100) }\end{array}$ & $\begin{array}{c}\text { Cronbach's } \\
\text { alpha }\end{array}$ & $\begin{array}{c}\text { Intraclass } \\
\text { correlation (n=27) }\end{array}$ \\
\hline NTM Symptoms & $75.7 \pm 17.9$ & 0 & $7 \pm 3.5$ & 0.82 & 0.79 \\
Body Image & $77.5 \pm 25$ & $2 \pm 1.0$ & $53 \pm 26.1$ & 0.74 & 0.72 \\
Digestive Symptoms & $78.7 \pm 15.3$ & 0 & $19 \pm 9.4$ & 0.67 & 0.74 \\
Eating Problems & $84 \pm 22.2$ & $2 \pm 1.0$ & $105 \pm 51.7$ & 0.89 & 0.94 \\
\hline
\end{tabular}

Data are presented as mean \pm SD. $n=203$. NTM: nontuberculous mycobacteria. NTM Module score range is $0-100$, where higher is better.

TABLE 4 Multivariable linear regression of factors associated with enrolment NTM Module scores

\begin{tabular}{|c|c|c|c|c|c|c|c|c|c|c|c|c|}
\hline & \multicolumn{3}{|c|}{ NTM Symptoms } & \multicolumn{3}{|c|}{ Body Image } & \multicolumn{3}{|c|}{ Digestive Symptoms } & \multicolumn{3}{|c|}{ Eating Problems } \\
\hline & Est & SE & p-value & Est & SE & p-value & Est & SE & p-value & Est & SE & p-value \\
\hline Intercept & 75.9 & 4.8 & $<0.0001$ & 84.4 & 6.6 & $<0.0001$ & 78.2 & 4.1 & $<0.0001$ & 89.6 & 5.6 & $<0.0001$ \\
\hline Male sex & 3.2 & 3.3 & 0.34 & -0.6 & 4.6 & 0.889 & 2.3 & 2.8 & 0.423 & 5.8 & 3.9 & 0.141 \\
\hline Bronchiectasis & 1.2 & 3.6 & 0.746 & 1.5 & 5.0 & 0.768 & 1.1 & 3.1 & 0.731 & 1.2 & 4.2 & 0.771 \\
\hline Treated COPD & -6.6 & 2.9 & 0.023 & -7.8 & 4.0 & 0.055 & -6.9 & 2.5 & 0.006 & -11.5 & 3.4 & 0.001 \\
\hline On treatment & 0.3 & 2.7 & 0.924 & 1.0 & 3.8 & 0.797 & 2.3 & 2.3 & 0.335 & -0.8 & 3.2 & 0.814 \\
\hline
\end{tabular}

n=203. NTM: nontuberculous mycobacteria; AFB: acid-fast bacillus. Est: predicted score lintercept+est: predicted score for patients with each characteristic). 
TABLE 5 Enrolment mean NTM Module scores for patients with pulmonary Mycobacterium avium complex (MAC) disease by patient disease characteristics and disease activity

\begin{tabular}{|c|c|c|c|c|c|c|}
\hline \multirow[t]{2}{*}{ NTM Module domain } & \multicolumn{3}{|c|}{ Subset with last AFB culture positive } & \multicolumn{3}{|c|}{ Subset defined by treatment and last AFB culture } \\
\hline & $\begin{array}{l}\text { Cavitary disease } \\
\text { (n=32] }\end{array}$ & $\begin{array}{l}\text { No cavitary disease } \\
(n=120)\end{array}$ & p-value & $\begin{array}{l}\text { Active MAC disease } \\
(n=43)\end{array}$ & $\begin{array}{l}\text { Prior MAC disease } \\
\qquad(\mathrm{n}=17)\end{array}$ & p-value \\
\hline Body Image & $68.3 \pm 33.0$ & $78.3 \pm 24.2$ & 0.12 & $72.2 \pm 25.0$ & $86.4 \pm 13.7$ & 0.007 \\
\hline Digestive Symptoms & $78.3 \pm 17.5$ & $78.4 \pm 15.2$ & 0.96 & $73.5 \pm 15.9$ & $80.2 \pm 11.1$ & 0.12 \\
\hline Eating Problems & $68.9 \pm 28.7$ & $86.7 \pm 20.4$ & 0.002 & $81.9 \pm 19.4$ & $96.7 \pm 6.5$ & $<0.0001$ \\
\hline
\end{tabular}

Data are presented as mean \pm SD. NTM: nontuberculous mycobacteria; AFB: acid-fast bacillus. Active MAC disease: never treated and last AFB sputum culture positive; Prior MAC disease: completed treatment and last AFB sputum culture negative. ${ }^{\#}$ : Chi-square or Fisher's exact test for categorical variables; t-test for comparison of continuous variables.

with Respiratory Symptoms scores increasing by $10.5(\mathrm{p}=0.006)$ and Vitality scores increasing by 10.2 $(\mathrm{p}=0.01$ ) in the starting-treatment group (figure $2 \mathrm{~b}$ ). Among on-treatment patients, no statistically significant improvement in HRQoL was observed. In the subset of 16 starting-treatment patients with poor scores at enrolment, NTM Symptoms scores improved by 15 points $(p=0.002)$. Respiratory Symptoms scores increased 16 points $(\mathrm{p}=0.02)$ in the 12 patients with poor scores at enrolment.

\section{Discussion}

Existing PRO tools, such as the QOL-B, measure respiratory symptoms and other effects of the disease on HRQoL (social functioning, emotional functioning) that may be applicable for patients with pulmonary MAC disease. To date, a validated PRO measure of NTM-specific symptoms has been lacking. The NTM Symptom Module performed well as a valid PRO for pulmonary MAC disease. Notably, NTM Symptoms scores increased 12 months after initiation of treatment, remaining stable in a comparison group who were already on treatment at enrolment. The Digestive Symptoms, Body Image and Eating Problems domains also demonstrated good psychometric properties; however, Body Image did not change with treatment. Across all domains, scores were lower/worse in patients with underlying COPD.

There is a lack of patient-focused outcome measures for NTM treatment trials. The small number of NTM clinical trials to date have used microbiological end-points as primary outcomes, with secondary outcomes of other measures of disease activity and HRQoL, such as the St George's Respiratory Questionnaire (SGRQ) and 6-min walk test [13]. Importantly, the NTM Symptom Module also demonstrated good psychometric properties in a recent clinical trial of patients with more advanced, treatment-refractory disease [14]. Measures of pulmonary function do not improve with NTM treatment, irrespective of underlying lung disease, and unlike Pseudomonas infection, NTM disease does not cause acute bronchiectasis or chronic

TABLE 6 Enrolment NTM Module and Quality of Life Questionnaire-Bronchiectasis (QOL-B) mean domain scores in treated pulmonary nontuberculous mycobacteria (NTM) patients

\begin{tabular}{|c|c|c|c|c|}
\hline & \multicolumn{2}{|c|}{ NTM Symptoms administered } & \multicolumn{2}{|c|}{ NTM Symptoms and QOL-B administered } \\
\hline & $\begin{array}{c}\text { Starting treatment } \\
\qquad(n=35)\end{array}$ & $\begin{array}{l}\text { On treatment } \\
(n=41)\end{array}$ & $\begin{array}{l}\text { Starting treatment } \\
\qquad(n=25)\end{array}$ & $\begin{array}{c}\text { On treatment } \\
(n=18)\end{array}$ \\
\hline NTM Symptoms & $73.9 \pm 16.7$ & $76.4 \pm 16.9$ & $72.9 \pm 14.7$ & $72.4 \pm 19.0$ \\
\hline Digestive Symptoms & $74.3 \pm 15.9$ & $81.1 \pm 15.1$ & $76.0 \pm 14.8$ & $79.6 \pm 18.8$ \\
\hline Eating Problems & $82.7 \pm 17.7$ & $85.9 \pm 17.2$ & $79.8 \pm 17.4$ & $84.0 \pm 21.0$ \\
\hline Body Image & $72.5 \pm 25.4$ & $78.0 \pm 23.2$ & $69.8 \pm 26.0$ & $75.8 \pm 25.7$ \\
\hline Respiratory Symptoms & & & $57.6 \pm 22.6$ & $73.1 \pm 18.4$ \\
\hline Physical Functioning & & & $40.3 \pm 32.4$ & $58.5 \pm 30.8$ \\
\hline Vitality & & & $38.7 \pm 24.0$ & $46.3 \pm 23.3$ \\
\hline Role Functioning & & & $55.5 \pm 29.7$ & $75.2 \pm 20.3$ \\
\hline Emotional Functioning & & & $75.3 \pm 17.9$ & $85.2 \pm 13.0$ \\
\hline Social Functioning & & & $53.9 \pm 29.4$ & $71.8 \pm 24.1$ \\
\hline Health Perceptions & & & $35.0 \pm 23.6$ & $49.1 \pm 19.2$ \\
\hline
\end{tabular}

Data are presented as mean \pm SD. Treatment burden not shown, too few to evaluate. 


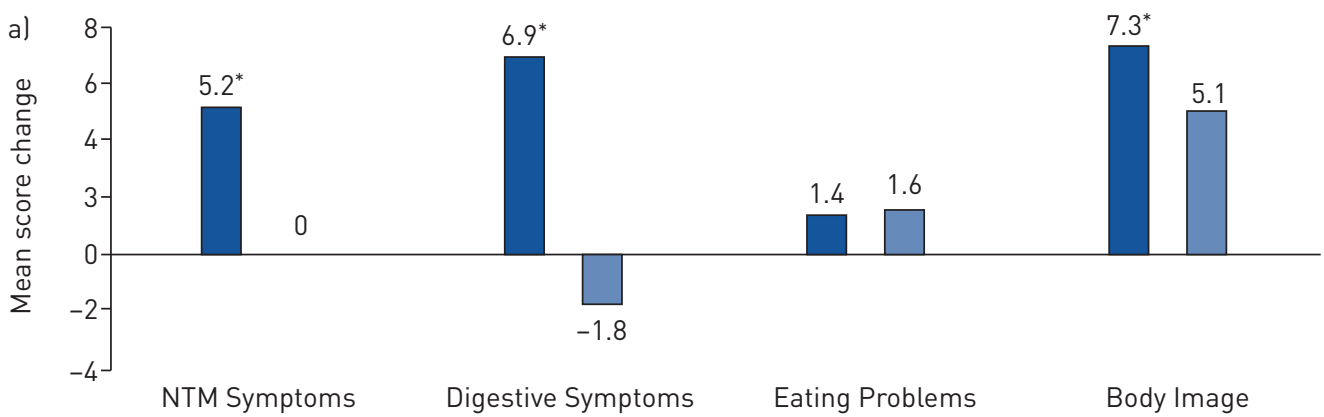

Starting treatment $n=25 \quad \square$ On treatment $n=18$



FIGURE 2 a) 12-month change in NTM Module domain scores in treated pulmonary Mycobacterium avium complex (MAC) patients ( $n=35$ starting treatment and $n=41$ on treatment), analysed using the paired t-test. b) 12-month change in NTM Module and Quality of Life Questionnaire-Bronchiectasis (QOL-B) domain scores in treated pulmonary MAC patients who completed both NTM Module and QOL-B ( $n=25$ starting treatment and $n=18$ on treatment), analysed using the paired t-test. NTM: nontuberculous mycobacteria; *: significant at $p<0.05$.

obstructive disease exacerbations [15]. In our study the NTM Symptoms score was not correlated with pulmonary function testing results, confirming its measurement of symptoms independent of lung function.

It is important to acknowledge that many NTM patients have underlying chronic, progressive bronchiectasis and/or obstructive disease that affects HRQoL [1, 2]. Several PRO measures of symptoms and functioning have been validated in populations with a variety of relevant lung diseases, including the SGRQ (COPD and bronchiectasis), Bronchiectasis Health Questionnaire (BHQ, bronchiectasis), QOL-B (bronchiectasis) and CFQ-R (cystic fibrosis). The SGRQ was developed without patient input and originally designed for COPD, but has since been validated for bronchiectasis [16]. Major limitations of the SGRQ include substantial response burden for patients, problematic recall windows (1-3 months), and no clear definition of a "respiratory attack", which is not common patient language. The recently developed BHQ is a composite measure of health status that may be useful for clinical practice but does not measure a clear construct [17].

The CFQ-R and QOL-B were developed by Quittner and colleagues using a similar methodology to the NTM Symptoms Module $[6,8,18]$. The QOL-B and CFQ-R both include Respiratory Symptoms and Physical Functioning domains that are relevant to pulmonary NTM disease. In our population, the QOL-B Respiratory Symptom score correlated with pulmonary function testing results, and was responsive to treatment for NTM disease. The CFQ-R includes 12 domains, and already incorporates Eating Problems, 
Body Image and Digestive Problems that were excluded from the QOL-B as not significant in the non-CF population, but included here for patients with NTM disease. The CFQ-R or QOL-B may be administered in conjunction with the NTM Symptom Module in patients with underlying bronchiectasis to fully evaluate the impact of NTM disease on HRQoL. None of the previously described PROs have been validated in NTM populations, though they may also be considered complementary measures.

In contrast, the NTM Symptom Module measures systemic, non-respiratory NTM-specific symptoms, including loss of appetite, feverishness or chills, bad taste in the mouth and problems with memory. This set of symptoms was identified using a detailed conceptual framework and input from patients and physician specialists in open-ended interviews and focus groups during development of the NTM Symptom Module [7]. Patient input is a key element of the 2008 FDA guidance on the development of PROs and formed the basis for the development of the NTM Symptom Module [5]. Further, the Digestive Symptoms, Body Image and Eating Problems domains were endorsed by patients as associated with NTM disease. NTM treatment is also commonly associated with gastrointestinal side effects [1]; despite this, we saw an improvement in Digestive Symptoms in patients treated for NTM disease.

Strengths of our study include the administration of the NTM Symptoms Module in a large, heterogeneous, representative population of pulmonary MAC patients. Overall, study patient characteristics were consistent with population-based data from Oregon with regards to age, sex and underlying bronchiectasis and COPD [2]. In addition, although the QOL-B was added to the study after initiation, resulting in a smaller sample size, we observed a significant improvement in key respiratory symptoms and functioning in MAC patients initiating treatment. Potential limitations include a lack of a standardised clinical evaluation and treatment regimen at enrolment. However, we were able to define subgroups representing a range of disease activity, and by including patients enrolled at a single centre primarily treated by two physicians there is likely less variation in routine clinical care. Additionally, we did not include objective measures of function (e.g. step count), symptoms (cough frequency), disease severity (global scale) or other PRO measures of HRQoL. As a result, we were unable to calculate an anchor-based minimal important difference.

The NTM Symptoms Module is a brief, easy to administer, disease-specific PRO developed following the 2009 FDA guidance [5]. The NTM Symptom Module assesses a unique set of NTM symptoms not captured in existing tools. Further longitudinal evaluation of the NTM Symptom Module is needed to establish the minimal important difference and evaluate the tool in patients with other NTM species and at different stages of disease.

Author contributions: E. Henkle had full access to all of the data in the study and takes responsibility for the integrity of the data and the accuracy of the data analysis. E. Henkle drafted the manuscript. G.P. Ranches, W. Plinke and H.K. Litvin contributed to data collection. K.L. Winthrop and A.L. Quittner contributed substantially to the study design, data analysis and interpretation, and the writing of the manuscript.

Conflict of interest: E. Henkle has nothing to disclose. K.L. Winthrop reports grants and personal fees from Insmed Incorporated (consultant honoraria), outside the submitted work. G.P. Ranches has nothing to disclose. W. Plinke has nothing to disclose. H.K. Litvin has nothing to disclose. A.L. Quittner reports grants from Insmed Incorporated, during the conduct of the study; non-financial support from Insmed (travel funds to present a poster), personal fees from Aradigm (PRO consulting) and personal fees from Bayer (PRO consulting), outside the submitted work.

Support statement: This work was supported by two grants from the ATS Foundation Research Program: a partnered ATS Foundation/American Lung Association of the Mountain Pacific Junior Investigator Award (E. Henkle) and the DeSouza Research Award (DS-311495, K.L. Winthrop). The development of the NTM Symptoms Module was supported by Insmed Inc. and Behavioral Health Systems (A.L. Quittner). Sponsors involved in the initial development of the NTM Module had no role in the design, data collection, analysis or interpretation of this study. Funding information for this article has been deposited with the Crossref Funder Registry.

\section{References}

1 Griffith DE, Aksamit T, Brown-Elliott BA, et al. An official ATS/IDSA statement: diagnosis, treatment, and prevention of nontuberculous mycobacterial diseases. Am J Respir Crit Care Med 2007; 175: 367-416.

2 Winthrop KL, McNelley E, Kendall B, et al. Pulmonary nontuberculous mycobacterial disease prevalence and clinical features: an emerging public health disease. Am J Respir Crit Care Med 2010; 182: 977-982.

3 Henkle E, Aksamit T, Barker A, et al. Patient-centered research priorities for pulmonary nontuberculous mycobacteria (NTM) infection. An NTM Research Consortium Workshop Report. Ann Am Thorac Soc 2016; 13: S379-S384

4 Retsch-Bogart GZ, Quittner AL, Gibson RL, et al. Efficacy and safety of inhaled aztreonam lysine for airway pseudomonas in cystic fibrosis. Chest 2009; 135: 1223-1232.

5 US Department of Health and Human Services, Food and Drug Administration. Guidance for Industry: Patient-Reported Outcome Measures: Use in Medical Product Development to Support Labeling Claims. 2009. Available from: www.fda.gov/regulatory-information/search-fda-guidance-documents/patient-reported-outcomemeasures-use-medical-product-development-support-labeling-claims Date last updated: 09 December 2016. 
6 Quittner AL, Buu A, Messer MA, et al. Development and validation of The Cystic Fibrosis Questionnaire in the United States: a health-related quality-of-life measure for cystic fibrosis. Chest 2005; 128: 2347-2354.

7 Quittner AL, Madan A, Saez-Flores E, et al. Development of a quality of life measure for nontuberculous mycobacteria (NTM) [abstract]. Eur Respir J 2015; 46: Suppl. 59, PA2635.

8 Quittner AL, O’Donnell AE, Salathe MA, et al. Quality of Life Questionnaire-Bronchiectasis: final psychometric analyses and determination of minimal important difference scores. Thorax 2015; 70: 12-20.

9 Streiner DL. Starting at the beginning: an introduction to coefficient alpha and internal consistency. J Pers Assess 2003; 80: 99-103.

10 Tavakol M, Dennick R. Making sense of Cronbach's alpha. Int J Med Educ 2011; 2: 53-55.

11 Qin S, Nelson L, McLeod L, et al. Assessing test-retest reliability of patient-reported outcome measures using intraclass correlation coefficients: recommendations for selecting and documenting the analytical formula. Qual Life Res 2019; 28: 1029-1033.

12 Shrout PE, Fleiss JL. Intraclass correlations: uses in assessing rater reliability. Psychol Bull 1979; 86: 420-428.

13 Olivier KN, Griffith DE, Eagle G, et al. Randomized trial of liposomal amikacin for inhalation in nontuberculous mycobacterial lung disease. Am J Respir Crit Care Med 2017; 195: 814-823.

14 Deshpande C, Quittner AL, Chou E, et al. Validation of the nontuberculous mycobacteria (NTM) module in NTM lung disease [abstract]. Eur Respir J 2018; 52: Suppl. 62, PA342.

15 Mehta M, Chapman KR, Heffer M, et al. Impact of pulmonary nontuberculous mycobacterial treatment on pulmonary function tests in patients with and without established obstructive lung disease. Respirology 2015; 20: 987-993.

16 Wilson CB, Jones PW, O'Leary CJ, et al. Validation of the St George's Respiratory Questionnaire in bronchiectasis. Am J Respir Crit Care Med 1997; 156: 536-541.

17 Spinou A, Siegert RJ, Guan WJ, et al. The development and validation of the Bronchiectasis Health Questionnaire. Eur Respir J 2017; 49: 1601532.

18 Quittner AL, Marciel KK, Salathe MA, et al. A preliminary quality of life questionnaire-bronchiectasis: a patient-reported outcome measure for bronchiectasis. Chest 2014; 146: 437-448. 